\title{
Immunocytochemical Traits of Type IV Fibrocytes and Their Possible Relations to Cochlear Function and Pathology
}

\author{
Joe C. Adams ${ }^{1}$ \\ ${ }^{1}$ Eaton-Peabody Laboratory, Massachusetts Eye and Ear Infirmary, 243 Charles Street, Boston, MA 02114, USA
}

Received: 29 August 2008; Accepted: 16 February 2009; Online publication: 10 March 2009

\begin{abstract}
One of the more consistent and least understood changes in the aging human cochlea is the progressive loss of fibrocytes within the spiral ligament. This report presents an animal model for type IV fibrocyte loss, along with immunocytochemical evidence that noise-induced loss of these cells may account for previously unexplained hearing losses. The remarkably low threshold for noise-induced loss of type IV fibrocytes, approximately $24 \mathrm{~dB}$ less than the threshold for adjacent hair cell destruction, may account for the prevalence of missing fibrocytes in humans. In mice, changes in the spectrum of traumatizing noise had little effect upon the site of loss of the fibrocytes, suggesting that the primary site of damage that induced the loss was the basal-most cochlear turn, a site expected to be damaged by all three noise bands. Type IV fibrocytes were found to immunostain for connective tissue growth factor (CTGF) and for transforming growth factor beta receptor 3 , a receptor that is known to activate CTGF expression. Type IV fibrocytes lack immunostaining for adenosine triphosphatase and connexins that are key players in potassium ion uptake and transmission, which suggests that they play little, if any, role in potassium recycling from perilymphatic space to the endolymphatic space. Consequently, their loss probably does not directly reduce this process. Immunostaining for a receptor for CTGF, low-density-lipoprotein-related protein 1, indicated that CTGF acts as an autocrine and a paracrine agent within the cochlea. The lack of CTGF paracrine effects following noise-induced loss
\end{abstract}

Correspondence to: Joe C. Adams - Eaton-Peabody Laboratory · Massachusetts Eye and Ear Infirmary - 243 Charles Street, Boston, MA 02114, USA. Telephone: +1-617-5733975; fax: +1-617-7200048; email: Joe_Adams@meei.harvard.edu of type IV fibrocytes may account for previously unexplained hearing losses.

Keywords: spiral ligament, hearing loss, cochlea, acoustic trauma

\section{INTRODUCTION}

Connective tissue cells of the spiral ligament (fibrocytes) constitute a significant proportion of cells within the entire cochlea but relatively little is understood regarding their functional significance. Morphological and cytochemical traits have led to the recognition of four types of fibrocytes (Spicer and Schulte 1991). The different fibrocyte types are found preferentially in different sites within the spiral ligament, but it is often necessary to employ electron microscopy or immunostaining to positively identify individual cell types. Among fibrocyte types, variable staining for transport-related enzymes left ambiguities regarding the identity and properties of type IV fibrocytes (Spicer and Schulte 1991). The report that all fibrocytes within the spiral ligament of rats are connected by gap junctions (Kikuchi et al. 1995), together with a variety of evidence from numerous laboratories, led to the realization that fibrocytes play key roles in $\mathrm{K}^{+}$ion uptake from perilymph and recycling of those ions back to endolymph. The reported gap junctional connectivity of all fibrocytes, together with imprecise information regarding cytochemical specializations of various cell types, has led to a common assumption that all fibrocytes within the ligament participate in $\mathrm{K}^{+}$ion recycling (e.g., Wang et al. 2002; $\mathrm{Qu}$ et al. 2007). Herein, evidence is presented that indicates that fibrocyte types III and 
IV probably have little to do with $\mathrm{K}^{+}$recycling. Instead, it appears that type IV fibrocytes may play a trophic role in the long-term viability of the cochlea.

Loss of fibrocytes from the human spiral ligament starts at a young age and progressively increases with time, but little is known about the functional consequences of the loss of the missing cells (Wright and Schuknecht 1972; Kusunoki et al. 2004). Loss or damage to spiral ligament cells following acoustic trauma in animals has been reported (Johnsson and Hawkins 1976; Liberman and Kiang 1978; Wang et al. 2002; Hirose and Liberman 2003; Ohlemiller and Gagnon 2007). The present report concerns loss of type IV fibrocytes in mice following noise exposure as a model for at least part of unexplained cell loss in the spiral ligament of humans. This work extends a previous study that showed type IV fibrocytes to have a surprisingly low threshold for noise-induced destruction (Wang et al. 2002). In the present report, cytochemical traits that are useful for distinguishing type IV fibrocytes from nearby cells and for revealing physiological traits of these cells are presented. These cells were found to exhibit much less immunoreactivity for $\mathrm{Na}^{+}, \mathrm{K}^{+}$-adenosine triphosphatase (ATPase) and for connexin 26 than nearby type II fibrocytes, indicating that they do not share type II fibrocytes' role in $\mathrm{K}^{+}$ion recycling. Instead, type IV fibrocytes were found to show immunostaining for the cytokine, connective tissue growth factor (CTGF), for an activator of CTGF expression (transforming growth factor beta receptor 3, TGF $\beta$ R3) and for the only known CTGF receptor. Other cochlear cells were also found to be immunoreactive for the receptor, suggesting that CTGF secreted by type IV fibrocytes acts in a paracrine fashion upon these other cochlear cells. These results suggest that the lack of CTGF produced by degeneration of type IV fibrocytes may have negative impacts upon those cells and lead to degradation of hearing.

Experiments were done to address questions raised by the study of Wang et al. (2002). That study reported two puzzling findings regarding noise-induced degeneration of type IV fibrocytes: the relatively low level of noise needed for their destruction and the location of the vulnerable cells. Following exposures to $8-16-\mathrm{kHz} 100-\mathrm{dB}$ noise, the mice had two loci of hair cell damage. There was a tonotopic locus, which corresponded to the expected site of maximal basilar membrane excursion produced by the noise and a more basal locus near the hook. The site of type IV fibrocyte destruction was situated between these two loci. This site of loss led Wang et al. to propose that damage of type IV fibrocytes was secondary to noise-induced excessive glutamate or $\mathrm{K}^{+}$ions that were released by the organ of Corti in the region between the two loci of hair cell loss. They suggested that fibrocytes may be vulnerable to high levels of $\mathrm{K}^{+}$ ions due to their reported expression of the combination of $\mathrm{Na}^{+}, \mathrm{K}^{+}$-ATPase and the cotransporter NKCC1. Vulnerability to glutamate toxicity was thought to be due to their expression of the glutamate transporter GLAST. Their assumptions regarding these specializations for $\mathrm{K}^{+}$ion and glutamate uptake now appear in doubt. Their explanation regarding the site of noise-induced type IV fibrocyte loss was tested in the present work by systematically changing the spectrum of the traumatizing noise.

The site of noise-induced maximum basilar membrane excursion was moved approximately one cochlear turn by altering the frequency content of the noise to determine how this would change the site of type IV fibrocyte loss. Interestingly, noise spectrum changes resulted in little change in the site of type IV fibrocyte loss. This finding suggests that organ of Corti damage near the hook, which has been reported to occur non-tonotopically in a number of species in response to a variety of traumatizing noises, is the dominant effect that induces type IV fibrocyte loss. The possibility is raised that long-term effects of type IV fibrocyte loss may contribute to progressive loss of hearing following acoustic trauma.

\section{MATERIALS AND METHODS}

Male CBA/CaJ mice (6-10 weeks old) were exposed to noise of different bandwidths. White noise was filtered through a Wavetek Brickwall filter (model 753A), amplified by a Crown amplifier (model 75A), and then led to a JBL compression driver (model $2446 \mathrm{H}$ ) for frequencies less than $16 \mathrm{kHz}$ or a Realistic tweeter (Radio Shack cat. 40-1377) for frequencies greater than $16 \mathrm{kHz}$. Three pass bands of noise were employed: 4-8, 8-16, and 20-40 kHz. Sound levels were measured with a calibrated 0.25-in. B \& K microphone. One day before the noise exposures, animals were sedated with Avertin $(250 \mathrm{mg} / \mathrm{kg}$, intraperitoneally, ip) and the right tympanum was obliterated with a tungsten needle with the visual aid of a Zeiss operating microscope. The purpose of this was to create a control ear with a conductive hearing loss for comparison with the other ear that was fully exposed to the noise. Eight control mice had a tympanectomy but were not exposed to noise to check for possible cytochemical effects of the tympanectomy alone. Noise exposures were for $2 \mathrm{~h}$ in a reverberant chamber that had no two sides parallel (Wang et al. 2002). The mice were placed in a cage made of wire hardware cloth and placed where the noise field was uniform within $1 \mathrm{~dB}$. Survival times following termination of the noise varied from $3 \mathrm{~h}$ to 6 days. At the designated survival time, the mice were 
anesthetized with urethane $(2.5 \mathrm{~g} / \mathrm{kg}$, ip) and exsanguinated by intracardial perfusion with phosphatebuffered saline (PBS, pH 7.3), followed by a fixative containing $10 \%$ formalin and $0.2 \%$ glutaraldehyde in PBS. The heads were placed in the fixative for $6 \mathrm{~h}$ at room temperature or overnight at $4^{\circ} \mathrm{C}$. The skull was then blocked with a razor blade to produce a specimen containing the two cochleas connected by the occipital bone with a flat surface in the horizontal plane made through the parietal bones to facilitate orienting the specimen when it was embedded in wax. The tissue was decalcified in $120 \mathrm{mM}$ ethylenediaminetetraacetic acid at room temperature and then embedded in low melting point Paraplast X-TRA (McCormick Scientific). Ten-micron serial sections were cut in the horizontal plane and collected on Super Frost Plus slides (Fisher Scientific). Staining for cell presence and condition was done with $0.1 \%$ Azure A in 0.1-M acetate buffer ( $\mathrm{pH} 3.1$ ). Immunostaining was performed with the following antibodies: rabbit anti-CTGF, Cell Sciences, Canton, MA, USA, CPC100; rabbit anti-CTGF, Abcam, Cambridge, MA, USA, ab6992; chicken anti-Na ${ }^{+}, \mathrm{K}^{+}$-ATPase, Cortex, San Leandro, CA, USA, CR2004C; mouse anti- $\mathrm{Na}^{+}, \mathrm{K}^{+}$, $2 \mathrm{Cl}^{-}$cotransporter, Developmental Studies Hybridoma Bank, University of Iowa, T4; rabbit anti-connexin 26, Zymed Labs, San Francisco, CA, USA, 512800; rabbit anti-TGFß R1, Santa Cruz sc-9048; rabbit anti-TGF $\beta$ R2, Santa Cruz sc-1700; goat anti-TGFß R3, Santa Cruz sc 6199; and rabbit anti-low-density-lipoprotein-related protein 1 (LRP1), Aviva Antibody Corp., ARP32793-P050. Gene expression within cochlear soft tissue for all the above-mentioned proteins was confirmed by an Affymetrix chip gene profile of mouse cochlear genes, which can be downloaded from the National Center for Biotechnology Information's Gene Expression Omnibus (http://www.ncbi.nlm.nih. gov/geo/query/acc.cgi?acc=GSE13421). An easily searchable version is available on line (http://re search.meei.harvard.edu/Otopathology/tbimages/ mouse.html). For immunostaining, slides were dewaxed in xylenes, hydrated through ethanols, and covered for $30 \mathrm{~min}$ in $5 \%$ normal horse serum (NHS) to block sites of non-specific antibody binding. Primary antibodies diluted in 1\% NHS as indicated in figure captions were applied and left overnight in a humidified chamber at room temperature. Secondary biotinylated antibodies (Jackson ImmunoResearch) appropriate for the primaries were applied for $1 \mathrm{~h}$, followed by avidin-biotin complex reagent (Vector Labs, Burlingame, CA, USA) for $1 \mathrm{~h}$ and then diaminobenzidine and $\mathrm{H}_{2} \mathrm{O}_{2}$ for $10 \mathrm{~min}$. In some cases, the detection system was enhanced using the biotinylated tyramine technique (Adams 1992). Washes in PBS were done between above-described steps.
Semiquantitative comparisons of effects of different frequency bands of traumatizing noise were made on four mice exposed to each noise band. Azure-stained sections were used to rate amounts of loss or damage of noise-induced type IV fibrocytes using the following scale: 0 was no apparent loss, 1 minimal loss, 2 moderate loss, and 3 severe loss. Slides were coded so that the rating was done with the observer blinded to the experimental condition. Scores were assigned to type IV fibrocyte regions in the basal-most three half turns and plotted on reconstructions of each case.

Micrographs were acquired with an Infinity $\mathrm{X}$ digital camera (Lumenera Corp.) on a Zeiss microscope with background subtraction applied to correct for illumination non-uniformities. The images were rendered monochromic, sharpened by unsharp masking, and gamma-corrected using Photoshop.

Experiments were carried out in accordance with the National Institute of Health Guide for the Care and Use of Laboratory Animals with the approval of the Infirmary Institutional Animal Care review board.

\section{RESULTS}

\section{Cytochemical characterization of type IV and related cells}

Previous descriptions of type IV fibrocytes characterized these cells based largely upon their being situated within the spiral ligament basal to the attachment point of the basilar membrane in gerbils (Spicer and Schulte 1991) and by the finding that, in CBA/CaJ mice, cells in that region were vulnerable to damage by acoustic trauma (Wang et al. 2002). As a means of identifying cells, cytochemical specializations are far more attractive than mere location because cytochemical features permit identification of particular cell types in regions where more than one cell type is present and because cytochemistry can help identify functional properties of cells. Accordingly, immunostaining for CTGF was found to be a useful trait for identification of type IV fibrocytes. Figure 1 shows enlarged views of the four half turns of a modiolar section immunostained for CTGF. Spiral ligament cells situated generally basal to the basilar membrane are positive for CTGF, with staining being limited largely to cells in the basal turn (Fig. 1C, D). In addition, root cells in the basal turn and Deiters cells in the second turn (Fig. 1A, B) are also immunopositive for CTGF. Results were the same with both of the two independent CTGF antibodies (not illustrated). Among type IV fibrocytes, those in the upper basal turn stained most robustly for CTGF. With increasing dilution of primary antibodies, staining intensity decreased, with staining of type IV fibrocytes 


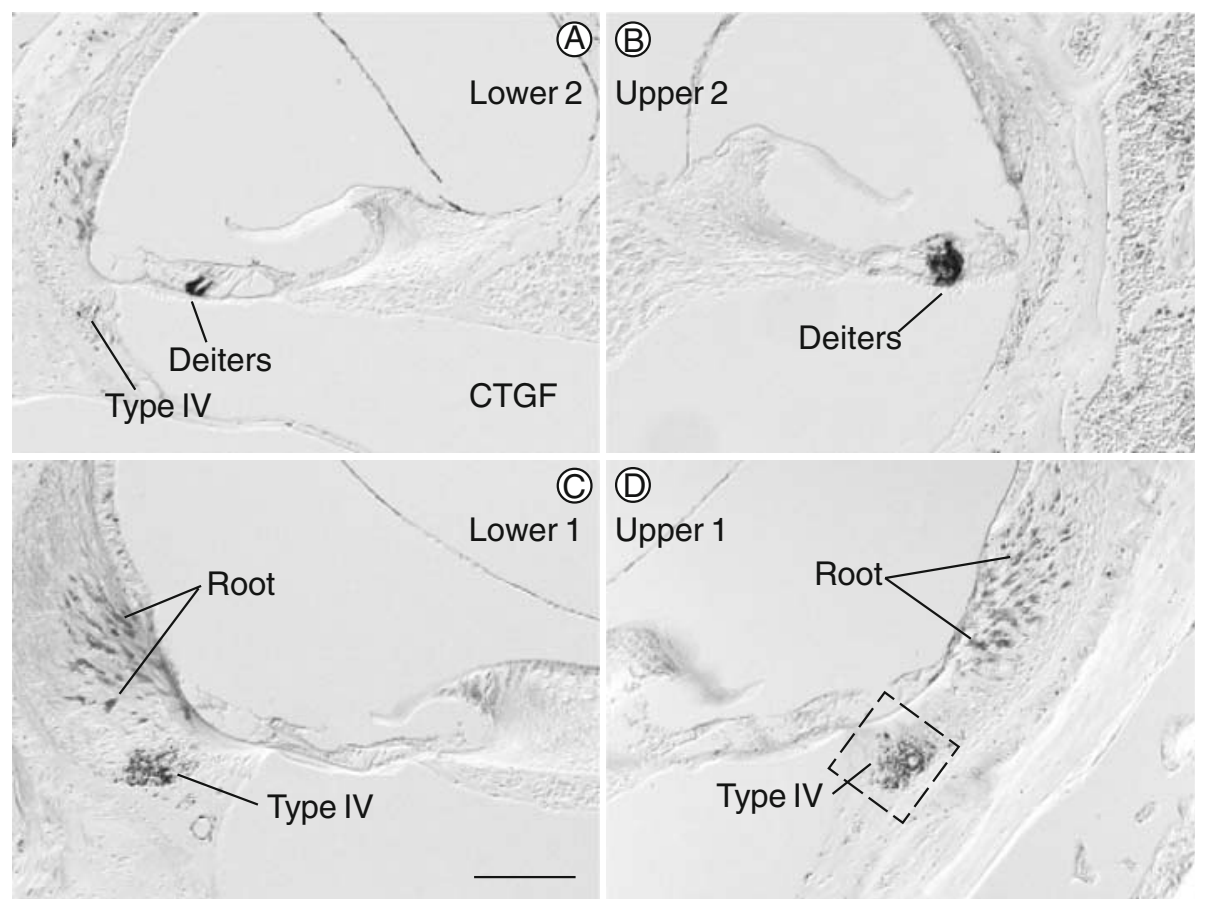

FIG. 1. A-D A control ear immunostained for CTGF (antibody concentration $1.1 \mu \mathrm{g} / \mathrm{mL}$ ) showing staining of type IV fibrocytes in the lower basal (lower 1) and upper basal (upper 1) half turns. Only a few faint cells are evident in the lower second (lower 2) and upper second (upper 2) half turns. Immunopositive root cells are conspicuous in the

being eliminated in the lower basal turn and in the second turn before that of fibrocytes in the upper basal turn. With increasing dilution of the primary antibody, staining for CTGF in all other cells types except for upper apical turn Deiters cells was also eliminated before that of upper basal type IV fibrocytes (not shown).

The finding that type IV fibrocytes were immunopositive for CTGF prompted a search for related compounds to support the CTGF findings and to better characterize the positive cells. Numerous reports in the literature that CTGF expression can be controlled by transforming growth factor beta (TGFß; e.g., Colwell et al. 2006) prompted investigation of TGF $\beta$ signaling in the cochlea. Antibodies against the receptors TGF $\beta 1 \mathrm{R}$, TGF $32 \mathrm{R}$, and TGF $\beta 3 \mathrm{R}$ were tried and only that against TGF $\beta 3 \mathrm{R}$ successfully stained the spiral ligament (Fig. 2A). Interestingly, root cells, which were positive for CTGF, were not positive for any of the TGF $\beta$ receptors, suggesting a lack of TGF $\beta$ control over CTGF levels in these cells. Deiters cells in the upper turn, a subset of fibrocytes in the spiral limbus, and scattered fibrocytes within the spiral ligament were also positive for TGFB3R (not shown). The locations of spiral ligament fibrocytes that immunostained for CTGF were also positive for TGFB-3R (Fig. 2B), suggesting that CTGF expression in type IV fibrocytes is under TGF $\beta$ control. basal turn and Deiters cells become increasingly darkly stained in the second turn. Nomarski optics. The dashed rectangle in panel D indicates the field shown in individual panels of Figures 3 and 4 . The calibration bar in panel $\mathbf{C}$ is $100 \mu \mathrm{m}$.

The finding that type IV fibrocytes were the only fibrocytes to stain for CTGF prompted a search for CTGF receptors that could reveal possibilities of cellcell communication between type IV and other cell classes. It has been shown that the LRP1 binds CTGF (Segarini et al. 2001). LRP1 appears to be the only CTGF receptor that has thus far been identified. Within the cochlea, a number of cell types were found to be immunopositive for LRP1, including type I fibrocytes, type IV fibrocytes, strial cells, cells in the spiral limbus, and spiral ganglion cells (Fig. 2G). Thus, CTGF, which is a secreted cytokine, appears to act both in an autocrine fashion upon type IV fibrocytes and in a paracrine fashion upon the other cells that are LRP1 positive.

Other cytochemical traits of type IV fibrocytes were found to be informative about their functions and to be useful for distinguishing them from nearby types II and III. Figure 2D shows that type IV fibrocytes immunostain for the $\mathrm{Na}^{+}, \mathrm{K}^{+}, 2 \mathrm{Cl}^{-}$cotransporter NKCC1 (SLC12A2), whereas they do not stain for $\mathrm{Na}^{+}, \mathrm{K}^{+}$-ATPase (Fig. 2C). This distinguishes them from nearby type II fibrocytes, which stain for both proteins (Fig. 2C, D). Figure 2E shows type III fibrocytes, which are situated immediately adjacent to the petrous bone and are immunopositive for aquaporin I, confirming a previous report (Stankovic et al. 1995). Note that there is spread of reaction 

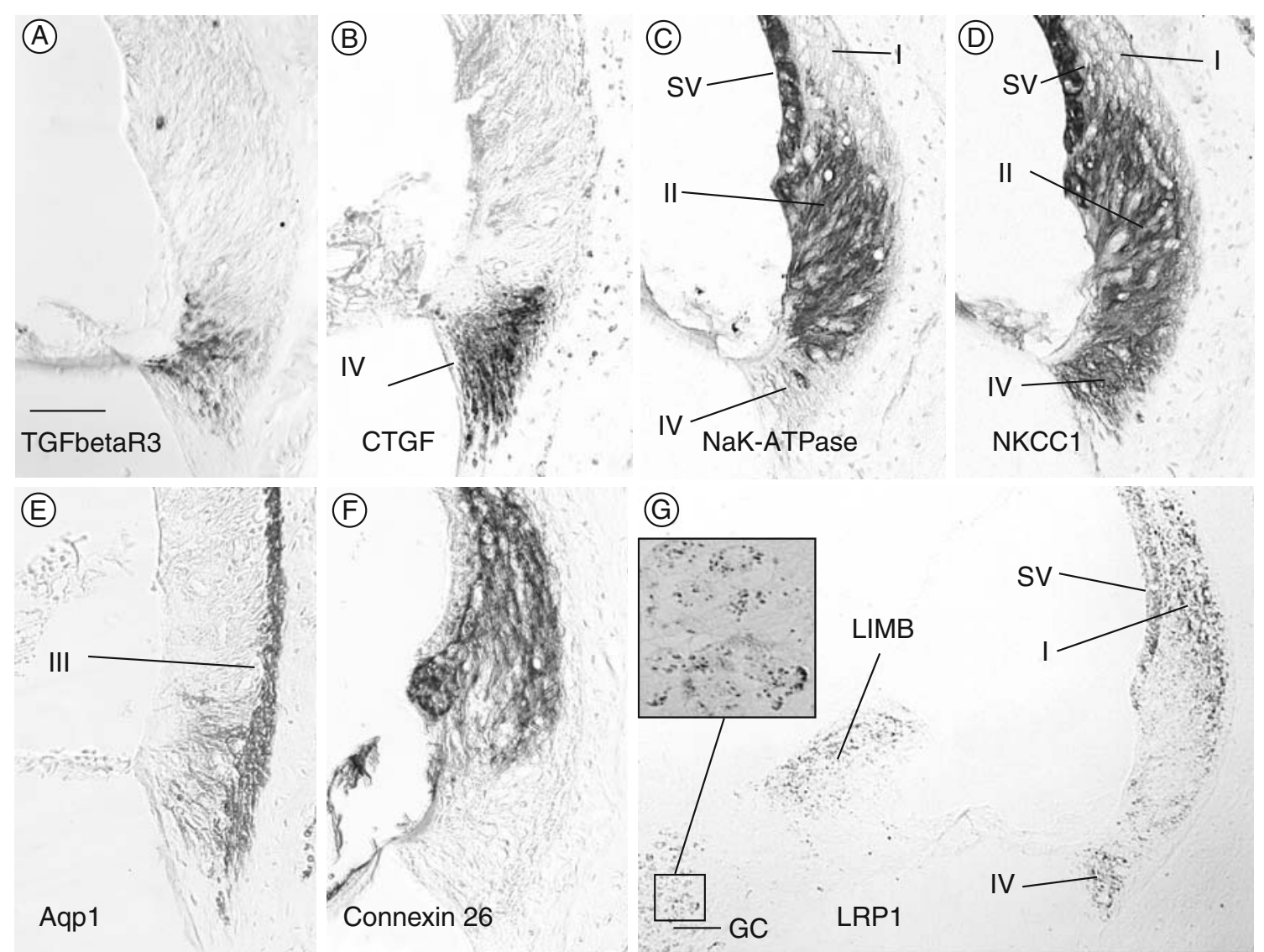

FIG. 2. Cytochemical traits of type IV fibrocytes and related cells. Panel A shows upper basal turn type IV fibrocytes staining for the TGF $\beta 3$ receptor (primary antibody concentration $0.2 \mu \mathrm{g} / \mathrm{mL}$, BT amplification). Panel B shows staining for CTGF $(1.1 \mu \mathrm{g} / \mathrm{mL})$ for comparison to panel A. Panel $\mathbf{C}$ shows staining for $\mathrm{Na}^{+}$and $\mathrm{K}^{+}$-ATPase (1:19 K dilution). The stria vascularis (SV) and type II fibrocytes (II) are darkly stained but only one cell in the region of type IV fibrocytes (IV) is positive, in contrast to NKCC1. Panel $\mathbf{D}$ shows staining for NKCC1 (1:9 K dilution) with a distribution very similar to that of $\mathrm{Na}^{+}, \mathrm{K}^{+}$ATPase with the exception that the type IV fibrocyte area is darkly stained. Panel $\mathbf{E}$ shows type III fibrocytes immunostained for aquaporin

$1(0.06 \mu \mathrm{g} / \mathrm{mL})$; type III fibrocytes are situated at the peripheral margin of the spiral ligament against the bone. There is staining within the type IV fibrocytes region but this appears to be processes of type III fibrocytes because no aquaporin-1-positive cells were ever found in this region. Panel $\mathbf{F}$ shows staining for connexin $26(0.56 \mu \mathrm{g} / \mathrm{mL})$. The region occupied by types III and IV fibrocytes are conspicuous by their lack of staining for connexin 26. Panel $\mathbf{G}$ shows staining for LRP1 $(0.28 \mu \mathrm{g} / \mathrm{mL}$, BT amplification) in a control ear. Types I and IV fibrocytes are positive, as well as stria vascularis, spiral limbus, and ganglion cells. The inset shows a higher magnification of ganglion cells. The calibration bar in A is $50 \mu \mathrm{m}$ and applies to panels A-F.

product into the region occupied by type IV fibrocytes. Examination of many sections stained for aquaporin I consistently failed to show immunopositive cell bodies in this region, suggesting that the spread of reaction product like that shown in Figure 2E may result from staining of processes of type III fibrocytes into this area. Finally, Figure 2F shows immunostaining for connexin 26. The remarkable feature of this image is the lack of positive staining of the type III and type IV fibrocytes, suggesting that they have few, if any, gap junctional connections with other fibrocytes.

\section{Effects of noise exposures}

Following noise exposures, decreases in immunostaining for CTGF were readily apparent, but the only cells that showed such changes were type IV fibrocytes.

Changes were most readily detected in the upper basal turn and these are compiled in Table 1. The greatest range of noise levels and survival times were done with noise exposures of $8-16 \mathrm{kHz}$. At $3 \mathrm{~h}$ following 92-dB noise exposures, three of four mice showed decreased staining for CTGF (indicated by the downward pointing arrows in the table). With the exception of the $6 \mathrm{~h}$ survival, where no change in staining was apparent, similar results were seen at 1-4day survivals. At 6 days of survival, no staining for CTGF was seen in the ears traumatized by $92-\mathrm{dB}$ noise (indicated by the $\mathrm{X}$ in Table 1). Accompanying this complete loss of immunostaining were overt changes in the appearance of type IV fibrocytes as shown by Azure staining. In all instances where CTGF immunostaining was reduced to nil, changes in cell morphology was evident in Azure-stained sections. In no case with abnormal Azure staining was immunos- 
taining for CTGF normal. The cases in which there were no signs of nuclear damage but there were decreases in immunostaining for CTGF (indicated by downward pointing arrows in Table 1) showed that immunostaining could be a more sensitive means of detecting acoustic trauma in type IV fibrocytes.

An example of reduced staining of type IV fibrocytes following noise exposures is shown in Figure 3A. This example is the only one out of six mice that were exposed to $110-\mathrm{dB} 4-8-\mathrm{kHz}$ noise and had a 4-day survival that did not show a change in staining of Azure-stained sections (not shown). The other five mice that had the same exposure and survival times as the one shown in Figure 3A showed changes like those in panels B and C. Panel B shows a complete lack of staining for CTGF in the exposed ear. Panel $\mathrm{C}$ shows a nearby section stained with Azure. A reduction in the cell density in the type IV fibrocyte area is apparent. This case is representative of all cases in which trauma was evident in Azure staining. That is, whenever Azure staining showed cell nuclei within the region indicated by the polygon in the control section adjacent to panel $\mathrm{C}$ that were shrunken, fragmented, or missing, nearby sections stained for CTGF either showed very little staining or, most often, no staining at all, as indicated by " $\mathrm{X}$ " in the table. Notice that the affected region (indicated by the polygon in the control section) does not include the area immediately adjacent to the bone (where type III fibrocytes are found) and that it extends superior to the attachment point of the basilar membrane. Thus, identifying type IV fibrocytes solely by a criterion of their being situated inferior to the basilar membrane attachment point would produce both false-positive and false-negative results.
Figure 4 shows the progression of changes in nuclei of type IV fibrocytes in the upper basal turn with increasing survival time following exposures to $96-\mathrm{dB}$ $8-16-\mathrm{kHz}$ noise. At 1 day postnoise, nuclei are rounded and slightly more darkly stained (arrowhead, panel A) in comparison to the contralateral control cells. At 2 days postnoise, mostly fragments remain in the type IV area (arrowheads in panel B). At 3 days postnoise, the center of the type IV area is clear of debris and cells (arrowhead, panel C). These results suggest that if noise trauma is sufficient to induce nuclear shrinkage in type IV fibrocytes, nuclear dissolution and cell death will follow.

No differences in immunostaining for CTGF were present between the two ears of the control mice with tympanectomies but no noise exposures. The tympanectomized ears of noise-exposed mice were therefore assumed to show the normal amount of CTGF immunoreactivity. All judgments of changes in immunostaining and Azure staining were made with respect to the control tympanectomized ear of given mice.

In contrast to noise-induced effects upon staining for CTGF, staining for TGFß3R was a less-sensitive indicator of damage. Staining for TGFB3R was eliminated in traumatized animals in which type IV fibrocytes were found to be missing, but, in short-term survival cases with 94- and 96-dB noise exposures, staining for TGF $3-3 R$ appeared the same in noiseexposed and control ears (not shown) when staining for CTGF was clearly reduced or eliminated.

The expression of genes that code for all proteins that are shown to be immunopositive in Figure 2 was confirmed by results of the Affymetrix chip analysis. They were scored present in all eight mice that were analyzed by the chip analysis.

TABLE 1

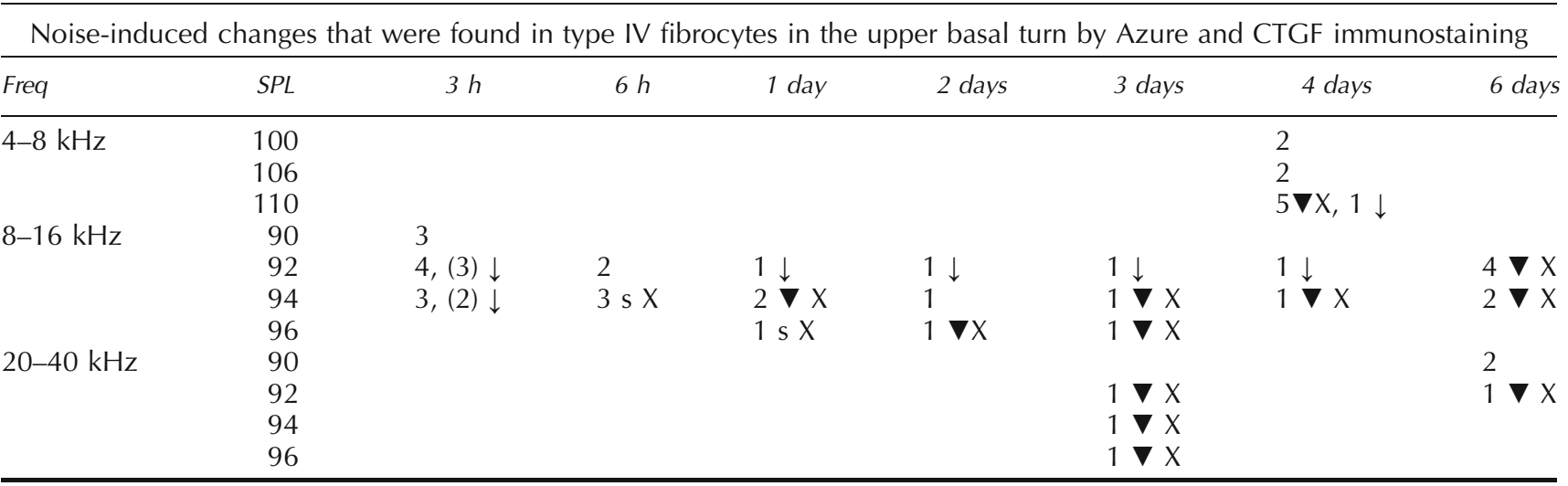

Column 1 indicates the noise bands employed. Column 2 indicates the sound pressure levels in decibel SPL. The cells in the remaining columns show the numbers of mice for the various conditions, along with indications of shrunken (indicated by "s") or fragmented or missing cells (triangles) as seen by Azure staining. Decreased staining for CTGF is indicated by downward pointing arrows. Areas that were totally devoid of immunostaining is indicated by " $\mathrm{X}$ ". In the 3 -h column, the numbers in parentheses indicate that three of the four mice exposed to 92-dB noise showed decreased staining and two of the three mice exposed to $94-\mathrm{dB}$ noise showed decreased staining 


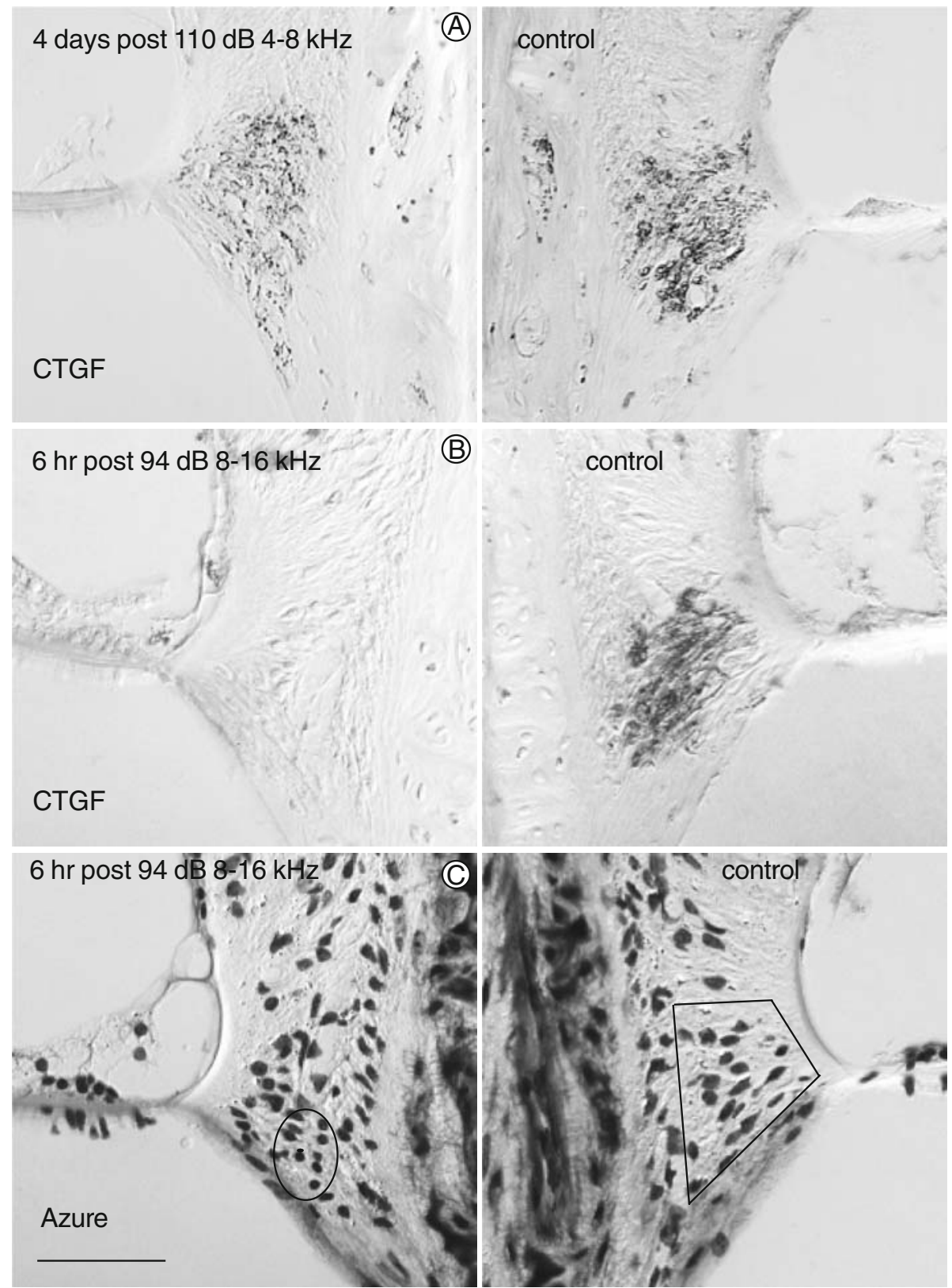

FIG. 3. A-C Extreme sensitivity of CTGF immunostaining to noise exposures (left column) as compared with matched control ears with conductive hearing loss (right column). All images are of upper basal turn. The top pair of images shows a dramatic decrease in staining in the left ear following a $110-\mathrm{dB} 4-8-\mathrm{kHz}$ exposure with a 4-day survival. No changes were evident in Azure-stained sections of this case (not shown).

\section{Effect of changing noise spectrum}

Wang et al. (2002) found that the site of noiseinduced type IV fibrocyte loss was complementary to the sites where inner hair cells were damaged and lost. The apical site of hair cell loss was the site of maximal excursion based upon the frequency map of basilar membrane motion. The basal site was near the hook region. The present experiments were done to move the site of maximal excursion by the traumatiz-
The middle pair of images shows that $6 \mathrm{~h}$ following a $94-\mathrm{dB} 8-16-\mathrm{kHz}$ exposure there was no staining apparent in type IV fibrocytes in the upper basal turn. The bottom pair of images are corresponding Azure-stained material of the same mouse shown in the middle panel. Only the earliest signs of noise-induced change are evident in the Azure-stained material (cells within the ellipse). The calibration bar is $50 \mu \mathrm{m}$.

ing noise band both apically and basally from place activated by the original $8-16-\mathrm{kHz}$ noise to see how that affected the locus of type IV fibrocyte loss. The best frequency of the basilar membrane in the mouse basal turn changes by approximately two octaves (Ehret 1983), so if the site of maximal excursion induced by the different noise bands were an important factor in determining the locus of type IV fibrocyte loss, changes in the locus of missing type IV fibrocytes would have been obvious. The striking 


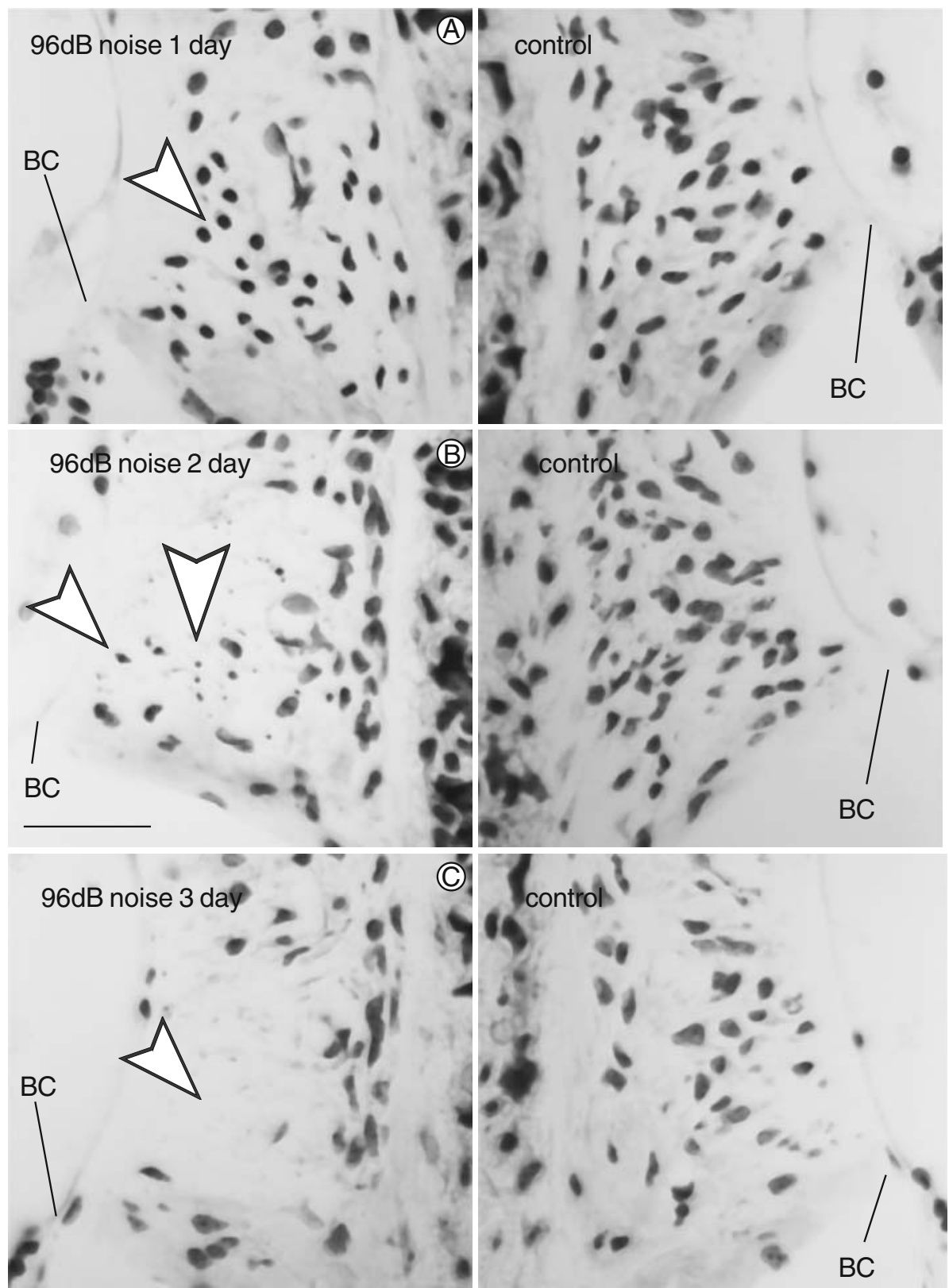

FIG. 4. Azure-stained sections of the type IV fibrocyte region of the upper basal turn. The dashed box in Figure 1D indicates the region covered by the micrographs of the present figure. The left column shows the range of cellular changes with increasing survival times following a $2-\mathrm{h}$ exposure to $96-\mathrm{dB} 8-16-\mathrm{kHz}$ noise to the left ears of three mice. The adjacent images in the right column show the

feature of the results of changing the noise spectra was that, despite changes in the noise frequency of more that two octaves, the loci of missing type IV fibrocytes remained with a region representing less than one octave of basilar membrane best frequency. Figure 5 shows representative cases for the different noise bands. In all cases, the sites of type IV cell loss remained in the upper basal turn irrespective of the frequency content of the traumatizing noise. The only consistent difference between the sites of cell loss for corresponding areas in the right ears, which had conductive hearing losses. The changes in cell morphology indicated by the arrowheads range from rounding and slight shrinkage of nuclei (panel $\mathbf{A}$ ) to small cellular debris (panel B) to missing cells (panel C). $B C$ indicates the basilar crest, the attachment point of the basilar membrane with the spiral ligament. The calibration bar in $\mathbf{B}$ is $15 \mu \mathrm{m}$.

the different frequency bands was that in none of the $20-40-\mathrm{kHz}$ cases was there evidence of type IV fibrocyte loss in the apical-most portions of the upper basal turn, as indicated by the arrows for the case illustrated in Figure 5. Another conspicuous difference between results with the three different noise bands was that the noise level required for inducing type IV cell loss with the $4-8-\mathrm{kHz}$ band was $18 \mathrm{~dB}$ greater than levels that were effective with the higherfrequency bands (Table 1). However, when the 4-8- 

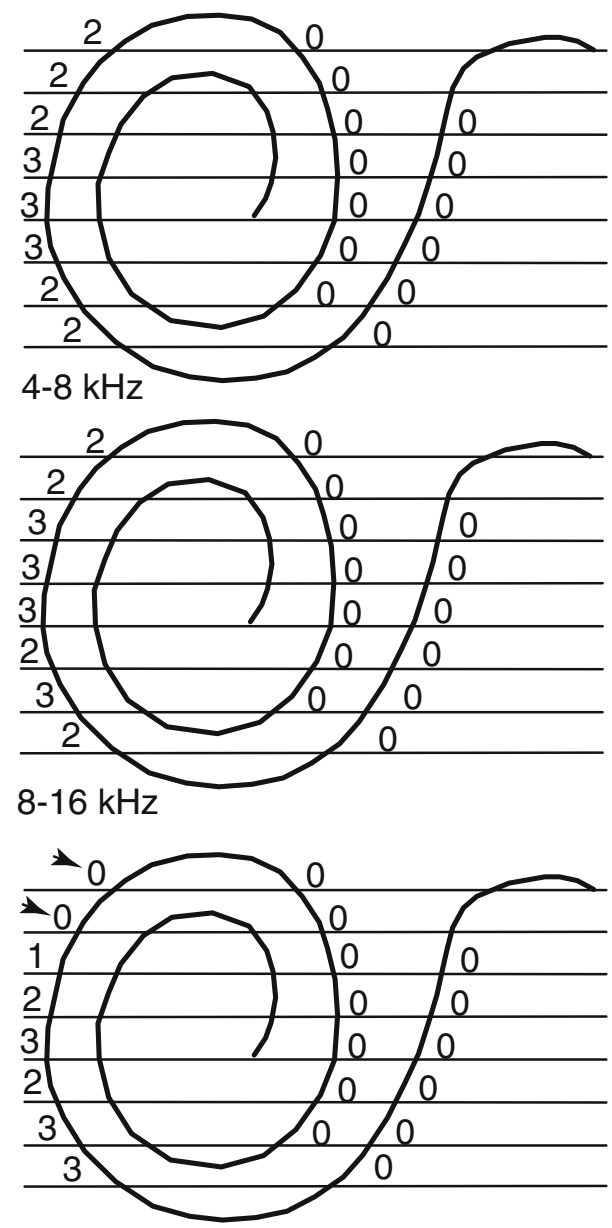

$20-40 \mathrm{kHz}$

FIG. 5. Representative plots of type IV fibrocyte loss produced by noise exposures with three different frequency bands. Thin horizontal lines represent sections through the cochlea spaced $100 \mu \mathrm{m}$ apart. The numbers indicate the degree of cell loss, with 0 being no cell loss and 3 being complete loss. There were no cell losses in the lower basal turn (farthest right) and lower second turn. No appreciable differences were found between mice exposed to 4-8and $8-12-\mathrm{kHz}$ noise bands. In none of the mice exposed to 20-40$\mathrm{kHz}$ noise were there missing cells in the basal-most upper basal turn (arrows in the lower figure).

$\mathrm{kHz}$ band was employed, the sites of cell loss did not move apical to the sites of cell loss that were seen when the $8-16-\mathrm{kHz}$ band was used.

\section{DISCUSSION}

The present results show a number of new features about type IV fibrocytes and related cells. Immunostaining showed these cells to be positive for the cytokine CTGF, for the TGF $\beta$ R3 receptor, which may control CTGF expression, and for LRP1, the only known CTGF receptor. Other cells, including type I fibrocytes, strial cells, spiral ganglion cells, and spiral limbal fibrocytes were also positive for LRP1, which suggests that CTGF secreted by type IV fibrocytes, in addition to autocrine effects, may also have widereaching paracrine effects upon other cochlear cells. Other cytochemical traits that distinguish type IV fibrocytes from nearby fibrocytes include their being positive for the cotransporter NKCC1, but not for $\mathrm{Na}^{+}$, $\mathrm{K}^{+}$-ATPase, and not being positive for aquaporin 1 or for connexin 26. Previous reports of type IV fibrocytes being highly vulnerable to acoustic trauma were extended to show that these cells situated in the upper basal turn were vulnerable to trauma by a wide range of frequencies, a finding which indicates that if type IV fibrocyte loss was secondary to organ of Corti damage, the primary sites of trauma were probably not the sites of maximal basilar membrane motion but rather the extreme base of the cochlea. The extreme base is the only site that would have been activated by all of the three noise bands that were used. Loss of staining for CTGF was found to be a far more sensitive indicator of noise-induced trauma than evidence of nuclear disruption or complete cell loss, as shown by conventional staining. Combined with the immunostaining results, analysis of the sites of missing or damaged cells following acoustic trauma in the upper basal turn showed that type IV fibrocytes are not confined to the region within the spiral ligament that is inferior to the attachment of the basilar membrane and that not all cells in that region are type IV fibrocytes. These findings demonstrate that the region in which type IV fibrocytes are present can be difficult to delineate without knowledge of cytochemical boundaries. The lack of staining for $\mathrm{Na}^{+}, \mathrm{K}^{+}$-ATPase and for connexin 26 indicated that type IV fibrocytes are probably not directly involved in $\mathrm{K}^{+}$uptake and recycling, as has been assumed. Altogether, these findings now permit ready identification of type IV fibrocytes, suggest that they signal other cochlear cells via paracrine release of CTGF, dispute previous assumptions regarding their function, and raise a number of possibilities regarding how their destruction might affect cochlear function. Questions are raised about the source of TGF $\beta$ that may upregulate CTGF expression in these cells, what conditions would prompt such signaling, and what the consequences of such signaling would be.

\section{Site of damage that provoked fibrocyte loss}

In a previous report, Wang et al. reported that the noise level that provoked upper basal turn type IV fibrocyte loss was considerably lower than the level that caused damage to sensory cells at the site of maximal basilar membrane excursion induced by the traumatizing noise but was close to the threshold for hair cell damage near the hook. This finding raised questions concerning the nature of the stress that 
provoked degeneration of the fibrocytes. Wang et al. reported that the locus of type IV fibrocyte loss was complementary to the two loci of inner hair cell damage, viz., it was between the tonotopic focus and the site of hair cell loss near the hook region. In the present work, despite changes in frequencies of the noise bands by more than two octaves, there was little evidence of corresponding changes in the sites of type IV fibrocyte loss. This finding, together with the fact that the threshold for hair cell damage near the hook is much closer to the threshold for type IV fibrocyte damage than was the threshold for hair cell damage at the tonotopic locus, indicates that the site of type IV fibrocyte loss is more likely to be related to damage to the organ of Corti near the hook rather than hair cell damage at the tonotopic focus of the noise band. That all three noise bands could damage hair cells near the hook is apparent from known properties of basilar membrane motion. The low pass nature of the lowfrequency slope of basilar membrane tuning would permit sound-induced motion of the hook region by all three noise bands. In contrast, the steep highfrequency slope of the basilar membrane filter function would prevent significant basilar membrane motion from being induced by the $20-40-\mathrm{kHz}$ noise at the more apical sites that were sites of maximum excursion in response to the lower-frequency noise bands. Consequently, all three noise bands utilized in the present study could have damaged the organ of Corti in the hook region. Tuning properties of basilar membrane motion are accurately reflected by tuning curves of auditory nerve fibers (Ruggero et al. 1997). The falloff in sensitivity at frequencies below $8 \mathrm{kHz}$ found in mouse high-frequency auditory nerve fibers (CF approximately $54 \mathrm{kHz}$, Taberner and Liberman 2005) predicts that high intensities of $4-8-\mathrm{kHz}$ noise would be required to induce motion in the hook region. The present finding that the threshold for type IV fibrocyte damage by $4-8-\mathrm{kHz}$ noise was much higher than the thresholds for the higher-frequency noises is in keeping with that prediction.

In assessing cellular damage induced by the noise exposures, Wang et al. found that, despite the fact that they were employing an optimal tissue processing protocol, they were unable to find morphological evidence for hair cell damage at any site following 94$\mathrm{dB}$ noise exposures, even though type IV fibrocytes were lost and increases in high-frequency-soundevoked thresholds were found at this noise level. The elevated thresholds indicated that there was damage to the tissue that could not be detected by light microscopy. It seems reasonable to assume that the loss of type IV fibrocytes was somehow induced by sound-induced damage to sensory cells but the finding that such sensory cell damage was not evident at sound levels that were adequate for type IV cell loss means that the nature of hair cell damage that provokes type IV cell loss will have to be sought using electron microscopy or perhaps by immunostaining for signs of hair cell damage. Such efforts were beyond the scope of the present study. The organ of Corti was examined for signs of damage but convincing signs were not found. These observations are not compelling, however, because tissue preservation was not optimal due to the tissue processing protocol having been optimized for immunostaining sensitivity. Nevertheless, the assumption that all three noise bands used in the present study may have damaged the lower basal turn organ of Corti seems reasonable, based upon considerations of basilar membrane mechanics discussed above and upon previous reports of hair cell loss near the hook in a number of species following noise exposures with a variety of noise types (Fried et al. 1976; Johnsson and Hawkins 1976; Liberman and Kiang 1978; Moody et al. 1978; Salvi et al. 1982; Wang et al. 2002; Harding et al. 2005).

Wang et al. argued that $\mathrm{K}^{+}$ions or glutamate released by the undamaged organ of Corti between the two sites of hair cell loss may have induced type IV fibrocyte loss. The present findings of a lack of immunostaining of type IV fibrocytes for $\mathrm{Na}^{+}, \mathrm{K}^{+}-$ ATPase indicates that these cells are not specialized for $\mathrm{K}^{+}$uptake and consequently the $\mathrm{K}^{+}$toxicity hypothesis of Wang et al. (2002) is not an attractive explanation of noise-induced type IV fibrocyte death. Similarly, the report that GLAST immunostaining in the spiral ligament of mouse does not include type IV fibrocytes (Jin et al. 2003) argues against the glutamate toxicity hypothesis. (The presence of GLAST was through the basis for presumed type IV cell glutamate toxicity.) The mechanisms of damage to the type IV fibrocytes remain unknown, just as the basis for the non-tonotopic-enhanced vulnerability of the organ of Corti in the base remains largely a matter of speculation.

The widely reported finding that the hair cells in the basal turn are more vulnerable to systemically administered ototoxic drugs suggests that basal turn cells are metabolically more vulnerable to stress than more apical ones. A metabolic vulnerability to chemical challenges could well be expected to extend to vulnerability to noise-induced stress, given that antiapoptotic and anti-oxidant treatments that prevent noise-induced hearing loss can also prevent damage by ototoxic drugs (reviewed by Henderson et al. 2006). It is interesting that protection from acoustic trauma, as afforded by systemic manipulations and by prior noise exposure, is effective at the tonotopic locus of the traumatizing noise but to a lesser extent or not at all, at the higher frequencies represented at the base of the cochlea (Wang et al. 2002; Yoshida et al. 1999; Yoshida and Liberman 2000). Unfortunately, 
in those studies, there were no observations concerning type IV fibrocytes. Nevertheless, those findings demonstrate that vulnerability of the basal organ of Corti to noise trauma is controlled by different processes than more apical sites. The present results indicate that vulnerability of type IV fibrocytes in the upper basal turn may be associated with damage to the lower basal turn organ of Corti. The molecular basis of this vulnerability can be realized only with the acquisition of much more knowledge concerning the molecular specializations of the cells associated with the vulnerabilities. It will be interesting to determine whether type IV fibrocytes share the lack of protective effects shown by the basal organ of Corti in response to treatments that protect tonotopic foci from acoustic trauma.

\section{Cytochemistry of type IV fibrocytes}

The cytochemistry of type IV fibrocytes reported here can offer suggestions concerning their vulnerability. Figure 2D shows that these cells immunostain for NKCC1. However, they were not positive for $\mathrm{Na}^{+}, \mathrm{K}^{+}$ATPase (Fig. 2C). Colocalization of these two proteins is a sign of a specialization for a highly efficient $\mathrm{K}^{+}$ uptake, for example, that shown by type II fibrocytes and by strial marginal cells (Fig. 2C, D). Type II fibrocytes being positive for NKCCl and $\mathrm{Na}^{+}, \mathrm{K}^{+}$ATPase have previously been reported (Schulte and Adams 1989; Crouch et al. 1997; Delpire et al. 1999). The NKCC1 protein is well recognized for its role in cell volume regulation in response to osmotic stress (e.g., Hoffmann and Dunham 1995). The presence of NKCC1 in type IV fibrocytes without colocalization with $\mathrm{Na}^{+}, \mathrm{K}^{+}$-ATPase suggests that these cells may be subject to osmotic stresses and that NKCG1 is expressed by these cells for coping with these stresses. How damage to the organ of Corti in the basal-most portion of the cochlea could induce osmotic or other stresses to cells in the upper basal turn is not immediately obvious. As was pointed out by Wang et al., it may be pertinent that the volume of the scala tympani in the hook region is much smaller than elsewhere in the cochlea due to the close proximity of the round window to the basilar membrane in that region. This reduced volume means that the scala tympani would be expected to have much less buffering capacity for ions or metabolites that may be spilled into perilymph by sound-induced damage of cells to the organ of Corti. The type IV fibrocytes that are most vulnerable to noise-induced damage are located apical to this region of reduced scala tympani volume and their vulnerability may arise from their proximity to this region.

A different cytochemical trait that type IV fibrocytes share with type III fibrocytes is a pronounced paucity of immunostaining for connexin 26 (Fig. 2F).
Similar findings have been reported for both connexin 26 and connexin 30 (Ahmad et al. 2003; Forge et al. 2003). Sparse staining for connexin 26 for cells in this region was also noted in the initial report on its localization in rat (Kikuchi et al. 1995). The sparse staining for gap junction proteins in type IV and III fibrocytes could hardly be otherwise because electron microscopy observations show that these cell types seldom contact one another or other cell types (personal unpublished observations). The sparse intercellular contact of types III and IV fibrocytes, together with their lack of the molecular bases for avid uptake of $\mathrm{K}^{+}$ions shown by type II fibrocytes, suggests that they have little, if any, direct role in $\mathrm{K}^{+}$ recycling that is attributed to types I and II fibrocytes. Consequently, it seems unlikely that if there are hearing deficits that result from type IV fibrocyte loss, they are not the results of deficits in $\mathrm{K}^{+}$recycling. The mild hearing losses reported by Wang et al. (2002) following 94-dB exposures, which destroyed type IV fibrocytes, support this view. It is further supported by results of Hirose and Liberman (2003) and by Ohlemiller and Gagnon (2007), who measured endolymphatic potentials following noise exposures that resulted in type IV fibrocyte loss and found no permanent loss of endolymphatic potential. It therefore can be safely concluded that type IV fibrocytes contribute little directly to $\mathrm{K}^{+}$recycling.

A recent report that rat type IV fibrocytes in culture show specializations that indicate their involvement in $\mathrm{K}^{+}$recycling ( $\mathrm{Qu}$ et al. 2007) appears to contradict the above conclusion. It may be that differences between that report and the present findings are due to species differences, but that assumption seems unlikely without evidence that cells equivalent to those collected by $\mathrm{Qu}$ et al. show different cytochemical traits from those described herein for type IV fibrocytes in situ.

\section{Implications of the findings}

In humans, degeneration of hair cells in the basalmost cochlea due to unknown causes begins at a very early age (Johnsson and Hawkins 1972) and progresses with increasing age (Bredberg 1968). This suggests that the basal organ of Corti in humans may be damaged by relatively mild noise exposures, as is the mouse ear (Wang et al. 2002). Such damage would not be detected by audiograms, which routinely test only as high as $8 \mathrm{kHz}$. Loss of the basal-most organ of Corti may, in turn, contribute to the loss of spiral ligament fibrocytes, which, in humans, begins in the first decade of life (Wright and Schuknecht 1972). Thus, in humans, the progressive loss of at least some spiral ligament fibrocytes may be a consequence of noise-induced damage. 
An example of pathology that may have been secondary to type IV fibrocyte loss was reported by Kujawa and Liberman (2006), who found that following $100-\mathrm{dB} 8-16-\mathrm{kHz}$ noise exposures in young mice there were long-term effects of more severe hearing losses than would be expected from comparable noise exposures later in life. Other than type IV fibrocyte loss, the only unusual histopathology that was found to be associated with this effect was enhanced loss of spiral ganglion cells in the old mice that had been traumatized at a young age. However, the ganglion cell loss could not account for the increased hearing loss. A lack of change in evoked acoustic emissions indicated that preneural effects were not the cause of the extra hearing loss. Therefore, the extra hearing loss was concluded to be a defect in neural processing. It may be that the observed loss of type IV fibrocytes contributed to the unusual loss of neural function through a lack of CTGF action upon the LRP1 receptors in the ganglion cells. LRP1 binds a variety of ligands, among which is CTGF (Segarini et al. 2001; Gao and Brigstock 2003; Shi-Wen et al. 2008). LRP1 has been reported to be associated with $N$-methyl-Daspartate receptors and PSD-95, both of which are present in spiral ganglion cells (Safieddine and Eybalin 1992; Kuriyama et al. 1993; Davies et al. 2001) and this association is believed to modulate synaptic transmission (May et al. 2004). Therefore, a chronic lack of CTGF might be expected to alter synaptic function. Further, LRP1 also plays a central role in the pathogenesis of Alzheimer's disease through its multiple roles in amyloid processing (Van Uden et al. 2000). CTGF has been found to play a neuroprotective role for retinal photoreceptors (Hauck et al. 2008; Harris-White and Frautschy 2005). The present results, together with above-cited references, suggest that CTGF may play a protective, or at least a trophic, role in the cochlea. It could play a role in auditory synaptic dysfunction or some other degraded neural function without neuronal loss due to loss of its action as an LRP1 ligand. Moreover, the presence of LRP1 in type I fibrocytes and in spiral limbus fibrocytes also opens the possibility that lack of CTGF that is normally secreted by type IV fibrocytes could lead to malfunction of these cells and this could also contribute to progressive hearing loss. It may be relevant in this context that, in a number of studies of animals with genetic predisposition to cochlear degeneration, missing type IV fibrocytes were found to be associated with deafness and premature cellular degeneration (Hequembourg and Liberman 2001; Ohlemiller and Gagnon 2004; Buckiova et al. 2006). Gradual progressive loss of hearing following acoustic trauma is common (see, e.g., Salvi et al. 1982) and the causes of this progression are not well understood. The present results suggest that loss of type IV fibrocytes could contribute in different ways to progressive posttrauma hearing loss.

CTGF has been studied extensively in the context of the role it plays in fibroblast biology and in extracellular matrix formation (Leask and Abraham 2003; Shi-Wen et al. 2008). It has been found to also play roles in diverse autocrine or paracrine actions upon other cell types, including vascular endothelial and smooth muscle cells, epithelial cells, neurons, glia, and bone cells (Moussad and Brigstock 2000; Brigstock 2003). Its function in the cochlea remains to be demonstrated, but the present finding of a CTGF receptor (LRP1) in a number of cochlear cells raises the possibility that it plays a paracrine role with LRP1immunopositive cells.

CTGF is a cytokine that has been widely studied in its role in connective tissue fibrosis but it is expressed in far more cell types than connective tissue and it has been shown to play roles in a wide variety of cell processes (Moussad and Brigstock 2000). The present study shows that it is normally present in type IV fibrocytes, root cells, and upper turn Deiters cells. (No changes in their immunoreactivity were observed in the latter two cell classes in the present experiments.) It seems unlikely that the normal role of CTGF in the cochlea is in fibrosis because fibrosis seldom occurs in the cochlea except as a postinflammatory response (Schuknecht 1993) and the fibrotic tissue appears in perilymphatic spaces, not in the spiral ligament. Instead, progressive loss of connective tissue cells in the spiral ligament is a common finding (Wright and Schuknecht 1972; Kusunoki et al. 2004). The present findings suggest that noise-induced loss of type IV fibrocytes may contribute to these losses and that previously unexplained hearing losses may arise from this process. This possibility is, of course, contingent upon the demonstration that the fibrocytes that are prone to degeneration in humans contain CTGF.

The present findings have implications for understanding human cochlear pathology but extending these findings to human ears awaits acquiring means of better identifying fibrocyte types in humans. In the present study, several antibodies were found to be useful for identifying type IV fibrocytes in mice. Unfortunately, these antibodies are most effective in tissue that is fixed in situ with a fixative that includes glutaraldehyde, a condition that is not possible for human tissue. Moreover, the unavoidable postmortem delay inherent in collecting human temporal bones presents a challenge for effective use of many antibodies, especially those directed against secreted proteins, and for pursuing implications of the present and other findings to humans. Perhaps pursuing questions raised by the present findings will lead to discovery of cytochemical traits of cochlear cells that can readily be identified in human tissue. 


\section{ACKNOWLEDGEMENTS}

This work was funded by NIH grant DC003929. Funding of the Affymetrix chip project was through the generosity of Mr. and Mrs. Charles Safford. The author is indebted to the unpublished findings of Dr. Michiko Node for prompting an interest in type IV fibrocytes. Technical help was provided by Sarah McCaffrey. Thanks go to Sarah McCaffrey, Jennifer O'Malley, Saumil Merchant, and Charles Liberman for comments and suggestions on the manuscript. The NKCC1 antibody developed by Dr. Lytle was obtained from the Developmental Studies Hybridoma Bank developed under the auspices of the NICHD and maintained by The University of Iowa, Department of Biology, Iowa City, IA 52242, USA.

\section{REFERENCES}

ADAMS JC. Biotin amplification of biotin and horseradish peroxidase signals in histochemical stains. J. Histochem. Cytochem. 40:1457-1463, 1992.

Ahmad S, Chen S, Sun J, Lin X. Connexins 26 and 30 are coassembled to form gap junctions in the cochlea of mice. Biochem. Biophys. Res. Commun. 307:362-368, 2003.

BREDBERG G. Cellular pattern and nerve supply of the human organ of Corti. Acta Otolaryngol. Suppl. 236:1-135, 1968.

BRIGSTOCK DR. The CCN family: a new stimulus package. J. Endocrinol. 178:169-175, 2003.

Buckiova D, Popelar J, Syka J. Collagen changes in the cochlea of aged Fischer 344 rats. Exp. Gerontol. 41:296-302, 2006.

Colwell AS, Krummel TM, Longaker MT, Lorenz HP. Fetal and adult fibroblasts have similar TGF-beta-mediated, Smad-dependent signaling pathways. Plast. Reconstr. Surg. 117:2277-2283, 2006.

Crouch JJ, Sakaguchi N, Lytle C, Schulte BA. Immunohistochemical localization of the Na-K-Cl co-transporter (NKCC1) in the gerbil inner ear. J. Histochem. Cytochem. 45:773-778, 1997.

Davies C, Tingley D, Kachar B, Wenthold RJ, Petralia RS. Distribution of members of the PSD-95 family of MAGUK proteins at the synaptic region of inner and outer hair cells of the guinea pig cochlea. Synapse 40:258-268, 2001.

Delpire E, Lu J, England R, Dull C, Thorne T. Deafness and imbalance associated with inactivation of the secretory Na-K-2Cl co-transporter. Nat. Genet. 22:192-195, 1999.

Eнret G. Peripheral anatomy and physiology II. In: Willott JF (ed) The Auditory Psychobiology of the Mouse. Springfield, Charles C. Thomas, pp. 169-200, 1983.

Forge A, Becker D, Casalotti S, Edwards J, Marziano N, Nevill G. Gap junctions in the inner ear: comparison of distribution patterns in different vertebrates and assessment of connexin composition in mammals. J. Comp. Neurol. 467:207-231, 2003.

Fried MP, Dudek SE, Bohne BA. Basal turn cochlear lesions following exposure to low-frequency noise. Trans. Sect. Otolaryngol. Am. Acad. Ophthalmol. Otolaryngol. 82:285-298, 1976.

GAO R, BRIGSTOCK DR. Low density lipoprotein receptor-related protein (LRP) is a heparin-dependent adhesion receptor for connective tissue growth factor (CTGF) in rat activated hepatic stellate cells. Hepatol. Res. 27:214-220, 2003.

HARDING GW, BoHNE BA, Vos JD. The effect of an age-related hearing loss gene (Ahl) on noise-induced hearing loss and cochlear damage from low-frequency noise. Hear. Res. 204:90-100, 2005.

HarRis-White ME, Frautschy SA. Low density lipoprotein receptorrelated proteins (LRPs), Alzheimer's and cognition. Curr. Drug Targets CNS Neuro. Disord. 4:469-480, 2005.
Hauck SM, Gloeckner CJ, Harley Me, Schoeffmann S, Boldt K, Ekstrom PA, Ueffing M. Identification of paracrine neuroprotective candidate proteins by a functional assay-driven proteomics approach. Mol. Cell. Proteomics 23:23, 2008.

Henderson D, Bielefeld EC, Harris KC, Hu BH. The role of oxidative stress in noise-induced hearing loss. Ear Hear. 27:119,2006

Hequembourg S, Liberman MC. Spiral ligament pathology: a major aspect of age-related cochlear degeneration in C57BL/6 mice. J. Assoc. Res. Otolaryngol. 2:118-129, 2001.

Hirose K, Liberman MC. Lateral wall histopathology and endocochlear potential in the noise-damaged mouse cochlea. J. Assoc. Res. Otolaryngol. 4:339-352, 2003.

Hoffmann EK, Dunham PB. Membrane mechanisms and intracellular signalling in cell volume regulation. Int. Rev. Cytol. 161:173262, 1995.

Jin ZH, Kikuchi T, Tanaka K, Kobayashi T. Expression of glutamate transporter GLAST in the developing mouse cochlea. Tohoku J. Exp. Med. 200:137-144, 2003.

Johnsson L-G, HAwkins JE. Sensory and neural degeneration with aging, as seen in microdissections of the human inner ear. Ann. Otol. 81:179-193, 1972.

Johnsson L-G, Hawkins JE. Degeneration patterns in human ears exposed to noise. Ann. Otol. Rhinol. Laryngol. 85(No.6):725739, 1976.

Kikuchi T, Kimura RS, Paul DL, Adams JC. Gap junctions in the rat cochlea: immunohistochemical and ultrastructural analysis. Anat. Embryol. (Berl.) 191:101-118, 1995.

KujaWa SG, Liberman MC. Acceleration of age-related hearing loss by early noise exposure: evidence of a misspent youth. J. Neurosci. 26:2115-2123, 2006.

Kuritama H, Albin RL, Altschuler RA. Expression of NMDAreceptor mRNA in the rat cochlea. Hear. Res. 69:215-220, 1993.

Kusunoki T, Cureoglu S, Schachern PA, Baba K, Kariya S, Paparella MM. Age-related histopathologic changes in the human cochlea: a temporal bone study. Otolaryngol. Head Neck Surg. 131:897903, 2004.

Leask A, Aвraham DJ. The role of connective tissue growth factor, a multifunctional matricellular protein, in fibroblast biology. Biochem. Cell. Biol. 81:355-363, 2003.

LibERMAN MC, KIANG NY. Acoustic trauma in cats. Cochlear pathology and auditory-nerve activity. Acta Otolaryngol. Suppl. 358:1-63, 1978.

May P, Rohlmann A, Bock HH, Zurhove K, Marth JD, Schomburg ED, Noebels JL, Beffert U, Sweatt JD, Weeber EJ, Herz J. Neuronal LRP1 functionally associates with postsynaptic proteins and is required for normal motor function in mice. Mol. Cell. Biol. 24:8872-8883, 2004.

Moody DB, Stebbins WC, Hawkins JE, JR, Johnsson LG. Hearing loss and cochlear pathology in the monkey (Macaca) following exposure to high levels of noise. Arch. Otorhinolaryngol. 220:47-72, 1978.

Moussad EE, BRIGSTOCK DR. Connective tissue growth factor: what's in a name? Mol. Genet. Metab. 71:276-292, 2000.

Ohlemiller KK, Gagnon PM. Cellular correlates of progressive hearing loss in 129S6/SvEv mice. J. Comp. Neurol. 469:377390, 2004.

Ohlemiller KK, Gagnon PM. Genetic dependence of cochlear cells and structures injured by noise. Hear. Res. 224:34-50, 2007.

Qu C, Liang F, Smythe NM, Schulte BA. Identification of ClC-2 and CIC-K2 chloride channels in cultured rat type IV spiral ligament fibrocytes. J. Assoc. Res. Otolaryngol. 8:205-219, 2007.

Ruggero MA, Rich NC, Recio A, Narayan SS, Robles L. Basilarmembrane responses to tones at the base of the chinchilla cochlea. J. Acoust. Soc. Am. 101:2151-2163, 1997.

SAFIEDDINE S, Eybalin M. Co-expression of NMDA and AMPA/kainate receptor mRNAs in cochlear neurones. Neuroreport 3:1145$1148,1992$. 
Salvi R, Perry J, Hamernik RP, Henderson D. Relationships between cochlear pathologies and auditory nerve and behavioral responses following acoustic trauma. In: Hamernik RP, Henderson D, Salvi R (eds) New Perspectives in Noise-Induced Hearing Loss. New York, Raven, pp. 165-188, 1982.

Schuknecht HF. Pathology of the Ear. Philadelphia, Lea \& Febiger, 1993.

Schulte BA, Adams JC. Distribution of immunoreactive $\mathrm{Na}^{+}, \mathrm{K}^{+}$-ATPase in gerbil cochlea. J. Histochem. Cytochem. 37:127-134, 1989.

Segarini PR, Nesbitt JE, Li D, Hays LG, Yates JR, 3Rd, Carmichael DF. The low density lipoprotein receptor-related protein/alpha2macroglobulin receptor is a receptor for connective tissue growth factor. J. Biol. Chem. 276:40659-40667, 2001.

Shi-Wen X, Leask A, Abraham D. Regulation and function of connective tissue growth factor/CCN2 in tissue repair, scarring and fibrosis. Cytokine Growth Factor Rev. 19:133-144, 2008.

Sitcer SS, Schulte BA. Differentiation of inner ear fibrocytes according to their ion transport related activity. Hear. Res. 56:53-64, 1991.
Stankovic KM, Adams JC, Brown D. Immunolocalization of aquaporin CHIP in the guinea pig inner ear. Am. J. Physiol. 269: C1450-C1456, 1995.

TABERNER AM, Liberman MC. Response properties of single auditory nerve fibers in the mouse. J Neurophysiol 93:557-569, 2005.

Van Uden E, Kang DE, Koo EH, Masliah E. LDL receptor-related protein (LRP) in Alzheimer's disease: towards a unified theory of pathogenesis. Microsc. Res. Tech. 50:268-272, 2000.

Wang Y, Hirose K, Liberman MC. Dynamics of noise-induced cellular injury and repair in the mouse cochlea. J. Assoc. Res. Otolaryngol. 3:248-268, 2002.

Wright JL, Schuknecht HF. Atrophy of the spiral ligament. Arch. Otolaryngol. 96:16-21, 1972.

Yoshida N, Liberman MC. Sound conditioning reduces noiseinduced permanent threshold shift in mice. Hear Res 148:213219, 2000.

Yoshida N, Kristiansen A, Liberman MC. Heat stress and protection from permanent acoustic injury in mice. J Neurosci 19:1011610124, 1999. 